\title{
Influence of ultra-processed foods consumption during pregnancy on baby's anthropometric measurements, from birth to the first year of life: a systematic review
}

Cristianny Miranda 1

(iD) https://orcid.org/0000-0002-6962-5784

Rafaela Cristina Vieira e Souza 2

(iD) https://orcid.org/0000-0003-2212-3776

Luana Caroline dos Santos 3

(iD) https://orcid.org/0000-0001-9836-3704

1-3 Escola de Enfermagem. Universidade Federal de Minas Gerais. Av. Professor Alfredo Balena, 190. sala 324. Belo Horizonte, MG, Brasil. CEP: $30130-100$. E-mail: cristiannyms@gmail.com

\begin{abstract}
Objectives: to perform a systematic review of studies that investigated the influence of ultra-processed foods (UPF) consumption during pregnancy on child's anthropometric parameters up to one year of life.

Methods: cohort and cross-sectional studies were researched in BVS, Cinahl, Cochrane, Embase, Pubmed, Scopus and Web of Science databases until March 2020, and the main descriptors were: "Pregnant Women", "Ultra-processed foods", "Birth Weight", "Small for Gestational Age", "Infant", "Newborn".

Results: seventeen articles were considered eligible and evaluated the associations between the exposures: ultra-processed dietary patterns; soft drinks, sugar-sweetened beverages, artificially sweetened beverages; fast food, junk food, sweets, snacks and the outcomes: birth weight and its classifications; length and head circumference at birth; birth weight adjustments according to gestational age; weight/age, length/age, body mass index/age and weight/length indices. The results showed: 36 non-significant associations between the exposures and the outcomes; 13 direct associations (outcomes versus ultraprocessed dietary patterns, soft drinks, artificially sweetened beverages, sweets, junk food) and 5 inverse associations (outcomes versus ultra-processed dietary patterns, soft drinks).

Conclusions: most of the evaluated literature did not demonstrate the influence of UPF consumption during pregnancy on the newborn's anthropometric measurements up to one year of life and denoted a smaller number of direct and inverse associations between the exposures and the outcomes.
\end{abstract}

Key words Ultra-processed foods, Pregnancy, Birth weight, Child, Food consumption

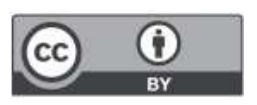




\section{Introduction}

Despite the importance of food for maternal and child's health, recent changes in the population's eating habits can be observed, mainly regarding the replacement of natural food (in natura and minimally processed foods) with ultra-processed foods which have high energy density and low nutritional quality. 1,2 This information is critically given that such replacements happen even during the gestational period, interfering with the nutritional status of the newborn, and later, of the child. 3

Pesquisa de Orçamentos Familiares (POF/2017$2018)^{4}$ (Research on Family Budget) recently highlighted a relevant increasing share of ultra-processed foods in the total calories determined by household food acquisition, from $12.6 \%$ in $2002-2003$ to $16.0 \%$ in 2008- 2009 and reaching $18.4 \%$ in $2017-2018$.

On this basis, maternal diet can influence the pre-gestational body mass index (BMI) and excessive gestational weight gain, besides being one of the main factors that interfere with pregnancy outcomes. The maternal metabolic profile may be damaged by an unhealthy diet (rich in saturated fats, sodium and sugar, and low in fiber, vitamins, and minerals), increasing oxidative stress and insulin resistance, and consequently, increasing fat and glucose transfer to the fetus. 5

Thus, abnormalities in fetal growth patterns can result in newborns small (SGA) or large for gestational age (LGA), with a great impact on the public health system - the high cost of medical and hospital expenses due to prolonged use of neonatal intensive care units, as well as a potential increase in neonatal and infant morbidity and mortality. ${ }^{6}$

The World Health Organization (WHO) considers SGA the newborns with a birth weight below the 10th percentile for gestational age and sex, and LGA those with a percentile higher than 90.7 It is estimated that babies are born SGA or LGA in $20 \%$ out of all births. 8

SGA newborns present a great risk of hypoxia during delivery, neonatal hypoglycemia, and necrotizing enterocolitis, a serious intestinal infection. Unlikely, the birth of LGA babies is associated with prolonged delivery, excessive maternal bleeding, severe vaginal ruptures, and cesarean section. Also, changes in growth, which start during the gestational period, can negatively affect the baby's health increasing the risk of future diabetes and cardiovascular diseases, for instance. 8

In this sense, the first postpartum year accounts for accelerated growth of the child and great biological vulnerability, due to the influence of factors such as birth conditions and extrauterine adaptation, socioeconomic conditions, access to healthcare, housing, sanitation, hygiene, and enough quality and quantity of food. Therefore, monitoring the child becomes essential in this age group, as studies have shown that linear growth deficits that can be fully recovered, begin around the $3^{\text {rd }}$ month of life and continues for two or three years. 9

Furthermore, this period is inserted in the first thousand days of the baby's life, which starts after conception until the age of two and represents a window of opportunities to improve the individuals health and to implement effective instruments to reduce malnutrition and contribute to the child's healthy growth and development, leading to positive impacts on the adult's health. 10

Studies have been carried out to clarify the association between the general quality of the diet and birth weight, and the risks of SGA and LGA, even so those associations are not well known. ${ }^{8}$ Besides, the influence of an unhealthy maternal diet composed of ultra-processed foods during pregnancy on the baby's weight gain is uncertain,11 there is still a lack of studies on topics like these in Brazil.

Therefore, considering the importance of assessing the baby's anthropometric measurements to promote child's health, and the increasing prevalence of ultra-processed foods consumption worldwide, this study aimed to conduct a systematic review of articles that investigated the influence of ultra-processed foods consumption during pregnancy on the anthropometric measurements of the baby's first year of life.

\section{Methods}

The present study is a systematic review of scientific articles that assess the association between the intake of ultra-processed foods during pregnancy and the baby's anthropometric measurements from birth to one year of life. As a strategy to elaborate the research question - "Is there any scientific evidence in the literature on the influence of ultra-processed foods intake during pregnancy, on the baby's anthropometric measurements from birth to one year of life?" - and to direct the bibliographic search, the anagram PECOS was used, which represents "population", "exposure", "comparison", "outcome" and "design". 12 In the present study, the population refers to the pregnant women, the exposure represents ultra-processed foods intake during pregnancy, the outcome analyzed is the baby's anthropometric measurements from birth to one year of life, and the design of the selected articles are transversal and 
longitudinal. The selection of the articles was carried out through bibliographic search in the BVS databases (Biblioteca Virtual em Saúde/Virtual Library in Health); Cinahl; Cochrane; Embase; Pubmed; Scopus and Web of Science. The terms used were related to the baby's anthropometric measurements (from birth to 1 year), to the ultra-processed foods intake, and to the period of interest - the pregnancy. The search strategy was composed of combinations of the following terms: (()(()(()("Pregnancy" [Mesh]) OR "Pregnant Women"[Mesh])) OR ("Pregnancy"[Title/Abstract] OR "Pregnant Women"[Title/Abstract]))))) $\quad$ AND ((("Ultraprocessed"[Title/Abstract] OR "Ultra-processed foods" [Title/Abstract] OR "ultra processed" [Title/Abstract] OR "ultraprocessed"[Title/Abstract] OR "ultra-processed"[Title/Abstract] OR "ready-toeat" [Title/Abstract] OR "ready-to-consume" [Title/Abstract] OR "industrialized foods" [Title/Abstract] OR "fast-food" [Title/Abstract] OR "fast food" [Title/Abstract] OR "fastfood" [Title/Abstract] OR "junk food" [Title/Abstract] OR "prepared food" [Title/Abstract] OR "candy" [Title/Abstract] OR "ice cream"[Title/Abstract] OR "chocolate" [Title/Abstract] OR "carbonated beverage" [Title/Abstract] OR "soft drink" [Title/Abstract] OR "sweetened beverage" [Title/Abstract] OR "snacks" [Title/Abstract] OR "Sausage" [Title/Abstract] OR "hot dog" [Title/Abstract] OR "Burger"[Title/Abstract] OR "dietary patterns" [Title/Abstract] OR "dietary behaviors" [Title/Abstract] OR "dietary habits" [Title/Abstract] OR "artificially sweetened beverages"[Title/Abstract] OR "cookie"[Title/Abstract] OR "salty snacks"[Title/Abstract] OR "chocolate drink mix" [Title/Abstract] OR "refined grains" [Title/Abstract] OR "sugar-sweetened beverages" [Title/Abstract] OR "ready-to-heat products" [Title/Abstract] OR "cake mixes" [Title/Abstract] OR "biscuits" [Title/Abstract] OR "chips" [Title/Abstract] OR "hamburguer"[Title/Abstract] OR "packaged soups" [Title/Abstract] OR "breakfast cereals" [Title/Abstract] OR "chicken nuggets" [Title/Abstract] $))))$ AND ((((()("Birth Weight" [Mesh]) OR "Infant, Small for Gestational Age"[Mesh]) OR "Infant, Newborn" [Mesh]) OR "Infant" [Mesh])) OR ("Birth Weight" [Title/Abstract] OR "Infant, Small for Gestational Age" [Title/Abstract] OR "Weight by Age" [Title/Abstract] OR "Body Weight" [Title/Abstract] OR "birth weight-for-length" [Title/Abstract] OR "birth weight for length" [Title/Abstract] OR "Weight-for-length" [Title/Abstract] OR "Weight for Length" [Title/Abstract] OR "weight-for-age"
[Title/Abstract] OR "Weight for age" [Title/Abstract] OR "length-for-age" [Title/Abstract] OR "Length for age" [Title/Abstract] OR "head circunference" OR "head circunference-for-age" [Title/Abstract] OR "head circunference for age" [Title/Abstract] OR "Large for gestational age"))))))). There were no restrictions on the period of time evaluated, but all works needed to be original studies conducted in human beings. The survey included every article published until March 2020. In addition to the electronic search, the reviewers also performed a manual analysis on the reference list for each study included, to identify those potentially relevant studies that were not found in the initial investigation.

Observational studies - cohort and crosssectional - published in Portuguese, English, and Spanish were selected for the present review. Eligibility criteria included studies with human beings that assessed the association between the ultra-processed foods intake (exposure) during the gestational period and the baby's anthropometric measurements (outcome) from birth to one year of life.

The exposure variable was the any ultraprocessed foods intake as defined in the NOVA classification. 2 Ultra-processed foods are industrial formulations entirely, or for the most part, produced from substances extracted from food (oils, fats, sugar, starch, proteins), derived from food constituents (hydrogenated fats, modified starch), or synthesized in a laboratory to provide products with attractive sensory properties. They are ready-to-eat or ready-to-heat foods, therefore, little or none culinary preparation is needed, making them accessible and convenient. They are usually combined with a sophisticated use of additives to make them durable and hyper-palatable. However, they have very low nutritional quality and tend to limit the in natura or minimally processed foods intake. ${ }^{2}$

As an exclusion criteria, for studies that assessed the ultra-processed foods intake in the form of dietary patterns, the pattern should mostly contain ultra-processed foods, as defined in the NOVA 2 classification. The articles that investigated food intake through food indexes (diet quality index) were not taken into consideration, as it would not be possible to discriminate the consumption of ultra-processed foods.

As for the outcomes, the baby's anthropometric measurements at birth and at any time until the end of the first year were evaluated: birth weight and its deviations - low birth weight $(<2,500 \mathrm{~g})^{13}$ and macrosomia (birth weight $>4,000 \mathrm{~g}$ ), 14 birth length and head circumference; weight/gestational age and their 
classifications - small/gestational age (SGA), appropriate/gestational age (AGA) and large/gestational age (LGA) and anthropometric indexes - weight/age (W/A), length/age (L/A), body mass index/age (BMI /A), and weight/length (W/L).

The articles found in the databases through the electronic search were stored in the EndNote ${ }^{\circledR}$ program to organize the references and eliminate duplicates. Then, two independent reviewers made the selection of initial articles after reading the titles, abstracts and keywords. After the initial selection of the articles, the Kappa test was performed to test the agreement between the evaluators, for which the statistical program, Statistical Package for the Social Sciences ${ }^{\circledR}$ (SPSS) version 19.0, was used. Byrt15 criteria were adopted to classify the result of the concordance test as: slight agreement: 0.21-0.40, fair agreement: $0.41-0.60$, good agreement: $0.61-0.80$ and very good agreement: $0.81-1.00$. The works selected in this stage were read in full and evaluated according to the eligibility criteria. A third reviewer judged whether the chosen articles would be eliminated or excluded in situations where the two reviewers disagreed.

The following data were extracted after the complete analysis of the selected articles: author, country and year of publication; study design; sample size (n) and maternal age (mean in years and standard deviation); method of assessing food consumption and exposure; assessed anthropometric baby's measurements and how these measurements were obtained - taken/self-reported; variables used to control confusion (adjustment variables) and main results.

The general and methodological quality of observational studies was assessed according to STROBE (Strengthening the Reporting of Observational Studies in Epidemiology). ${ }^{6}$ The maximum score that can be achieved in this assessment is 22 points, of which 9 points refer to the section "methods" of the studies.

\section{Results}

A total of 1551 articles was found, and after removing duplicates ( $\mathrm{n}=509), 1042$ titles, abstracts, and keywords remained to be analyzed (Figure 1). The Kappa concordance index found was 0.731 , indicating good agreement. 15 Having read the titles, abstracts, and keywords and keeping the eligibility criteria, the reviewers excluded 986 articles, leaving 56 to be read in full (Figure 1).

After the full reading, the reviewer 1 excluded 31 articles, and the reviewer 2 excluded 41 articles.
The two reviewers agreed on the selection of 11 studies and disagreed over 18. A third reviewer judged the relevance of the 18 articles on which the two previous reviewers disagreed and decided to exclude 12 of them. Thus, 17 studies were considered eligible for the current review. Figure 1 displays the reasons for exclusions.

Regarding the quality of the studies, 16 the total average and the "Methods" section got 17.26 (SD = $1.76)$ and $7.01(\mathrm{SD}=0.58)$ points, respectively.

Table 1 presents the main characteristics and results of the studies assessed, which were published between 1995 and 2019.

Among the selected articles, five were conducted in the United States, 17-21 two in Norway, 8,22 two in Australia, 23,11 and one in Germany, 24 one in Spain, 25 one in Ghana,26 one in Canada, 27 one in Netherlands, 28 one in New Zealand,29 one in England 30 and one in Brazil5 (Table 1).

Most of the studies were of prospective cohort design $(n=13) 5,8,11,17-22,24,27,28,30$ and four were cross-sectional.23,25,26,29 The sample size ranged from 12725 to 65,9048 women, with seven studies presenting over 1,000 participants $8,18,22,24,27,29,30$ (Table 1).

Maternal age was not presented in most part of the articles $(n=11)$. In the others, there was a variation from 24.20 (SD 5.40) years21 to 32.50 (SD 4.60) years 27 (Table 1).

It is noteworthy that in eleven $5,8,11,17,19,22,24,26,27-$ 29 of the seventeen articles selected for this review, the mothers in the sample presented some comorbidity before or during pregnancy, such as gestational diabetes, hypertension (including preeclampsia), overweight/obesity, depression, eating disorders (nausea during pregnancy), and chronic illnesses such as chronic hypertension, kidney diseases, and systemic lupus erythematosus. Out of the twelve studies, two were cross-sectional26,29 and the others were cohort (Table 1).

Besides the use of these variables (maternal comorbidities) in the adjustment of the multivariate model, other treatments were used to remove confounding factors related to these comorbidities such as analysis of variance and chi-square tests, 28 or multivariate analyses 26 to verify whether there was an association between maternal comorbidities and dietary patterns. In Alves-Santos et al. research, 5 a direct acyclic graph used for each outcome was developed to identify a minimal yet sufficient set of covariates to remove confusion from the analysis statistic. Only two studies 19,24 lack information on how these maternal comorbidities variables were considered. 


\section{Figure 1}

Flow chart illustrating the selection of the articles used for the present systematic review about the association between the consumption of ultra-processed foods during pregnancy and the baby's anthropometric measurements from birth to one year of life.

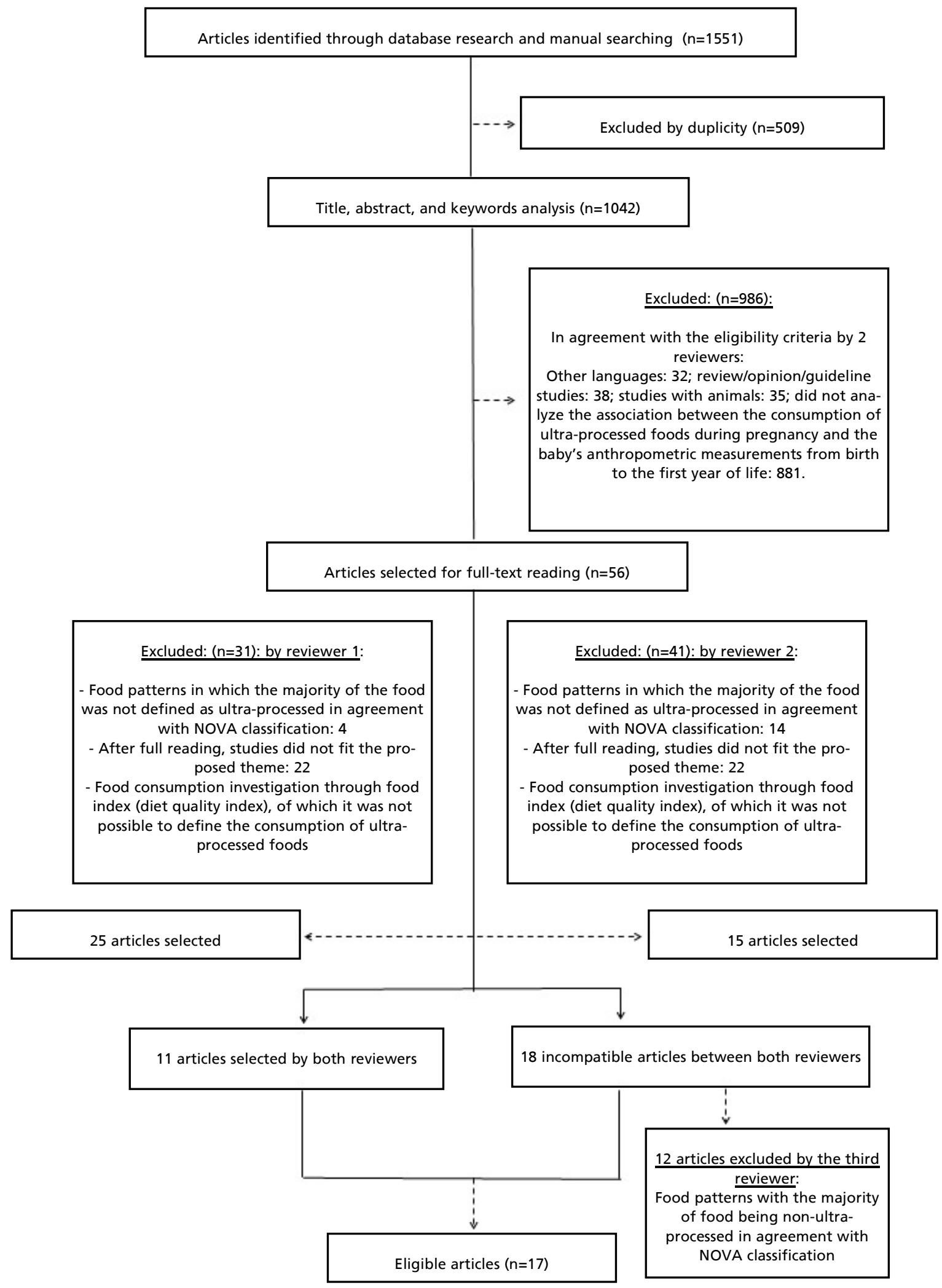


Food consumption was investigated using mostly the food frequency questionnaire (FFQ). Sixteen studies $5,8,11,17,18,20-30$ used this instrument, and one opted for a questionnaire prepared by the authors themselves 19 (Table 1). In the latter, Moss and Harris 19 prepared a questionnaire to assess the weekly intake of fast food, including the frequency of consumption in typical fast-food chains like McDonald's and Kentucky Fried Chicken.

The moment to assess food consumption through the FFQ assigned to mothers varied from the first to the third trimester of pregnancy8,11,17,18,20,22,24$27,29,30$ or it was considered the previous months to pregnancy $5,19,23,28$ and in one study, this period was not mentioned. 21

Regarding the analysis of food consumption (exposure), ten studies carried out using dietary patterns, mostly composed of ultra-processed foods, $5,8,17,18,21,23,26,28-30$ five for beverages such as soft drinks, sugar-sweetened beverages (regular pops or soft drinks, and sugar or honey added to tea or coffee) or artificially sweetened beverages (diet soft drinks, soft drinks and artificial sweetener added to tea or coffee) $20,22,24,25,27$ and four through investigation on the consumption of "fast food", "junk food" (soft drinks, fast food and/or processed meats and chips) and specific foods such as sweets and snacks. $11,19,20,24$

In the studies that evaluated dietary patterns, the authors described the patterns and wrote phrases indicating that the foods were ultra-processed: "fast food (rich in saturated fat and energy)" 5,17; "snacks with high sugar/energy content" 26; "Processed foods low in nutrients and dense in energy, high in saturated and trans fats, sodium and refined sugars"18; "Junkfood (unhealthy, energy-dense, low-nutrient diet) associated with increased levels of obesity"29; "Processed foods with a high-fat content"30; "Foods rich in calories and low in nutrients, high in sodium and sugar". ${ }^{21}$ Also, foods such as cakes, sweet pies, and sweets are generally considered to be ultraprocessed foods, as noted in the Pesquisa de Orçamentos Familiares, ${ }^{4}$ keeping in mind that homemade sweets and cakes are consumed by a smaller portion of the population. 4

The ultra-processed groups included: "high Western" pattern - salty and sweet snacks, desserts, processed meat, sweetened beverages, french fries 8 ; "fast food and candies" pattern - fast food and snacks, cakes, cookies, sweets or desserts5; "latent class1" pattern - processed meats, french fries, sweets, salty snacks and soft drinks 17; "non-health conscious diet" pattern - sweetened drink, ice cream, chocolate energy drink, milk drinks and soda26; "processed" pattern - processed meat, fast food, snacks, sweets and soft drinks 18 and "highfat/sugar/takeaway" pattern - ready-to-eat foods, potato chips. 23

Other ultra-processed patterns included: "energy-rich dietary pattern" - breakfast cereals, margarine, snacks/sweets 28 ; "junk food" pattern - ice cream, cookies, cakes, sweetened cereal, crisps, chocolate bars and chocolate energy drink 29 ; "processed" pattern - sausages and burgers, chips and crisps and "confectionery" pattern - confectionery, chocolate, sweets, cookies, cakes 30 and "Nutrient Dilute" pattern - salty snacks, cakes, cookies, pastries, gelatine dessert and ice cream. ${ }^{21}$

The most investigated anthropometric measurements in the studies were those at birth, such as birth weight. $8,18,19,21,22,24,26,28,30$ Other authors prioritized macrosomia11,20,22,24 and adjustments of the gestational weight/age index - SGA 8,23,24,25,29 and LGA. 5,8,20,24 In thirteen studies, the measurements were taken - in eleven, they were obtained from hospital records $5,8,11,17,18,22,24,26,28-30$ and in two20,27 they were taken by the researchers; in three studies they were self-reported by the mothers $19,21,25$ and in one study, 23 , such information was not mentioned (Table 1).

The direct association between the consumption of ultra-processed foods and the baby's anthropometric measurements were found in six studies of this present review. $5,8,11,20,25,27$

Englund-Ogge et al. 8 identified that the "high prudent" pattern (composed of vegetables, fruits and whole grains) was associated with the lowest birth weight $(\beta=-0.041$; CI95\% $=-0.068--0.013)$ and with fewer chances of LGA $(\mathrm{OR}=0.84$; CI95\% $=0.75-0.94)$ when compared to the "high Western" pattern. On the other hand, in the study by Alves-Santos et al.5 the "fast food and candies" pattern was associated with a greater chance of LGA babies $(\mathrm{OR}=4.38 ; \mathrm{CI} 95 \%=1.32-14.48)$ and with length at birth $>$ percentile $90 \quad(\mathrm{OR}=4.81$; CI95\%=1.77-13.07). Both studies had a longitudinal design (prospective cohort; Table 1).

In a cross-sectional evaluation, Gomez Roig et al. 25 verified a higher consumption of Coke among mothers in the SGA group ( $p=0.004)$, while mothers in the AGA group drank more diet Coke $(p=0.03)$. Among mothers of normal weight, Phelan et al.20 reported in a prospective cohort, a higher birth W/A of the baby $(\beta=0.16 ; p=0.04)$ in those mothers with a higher intake of soft drinks during pregnancy (Table 1).

The cohort study by Azad et al.27 described a direct association between the daily intake of artifi- 


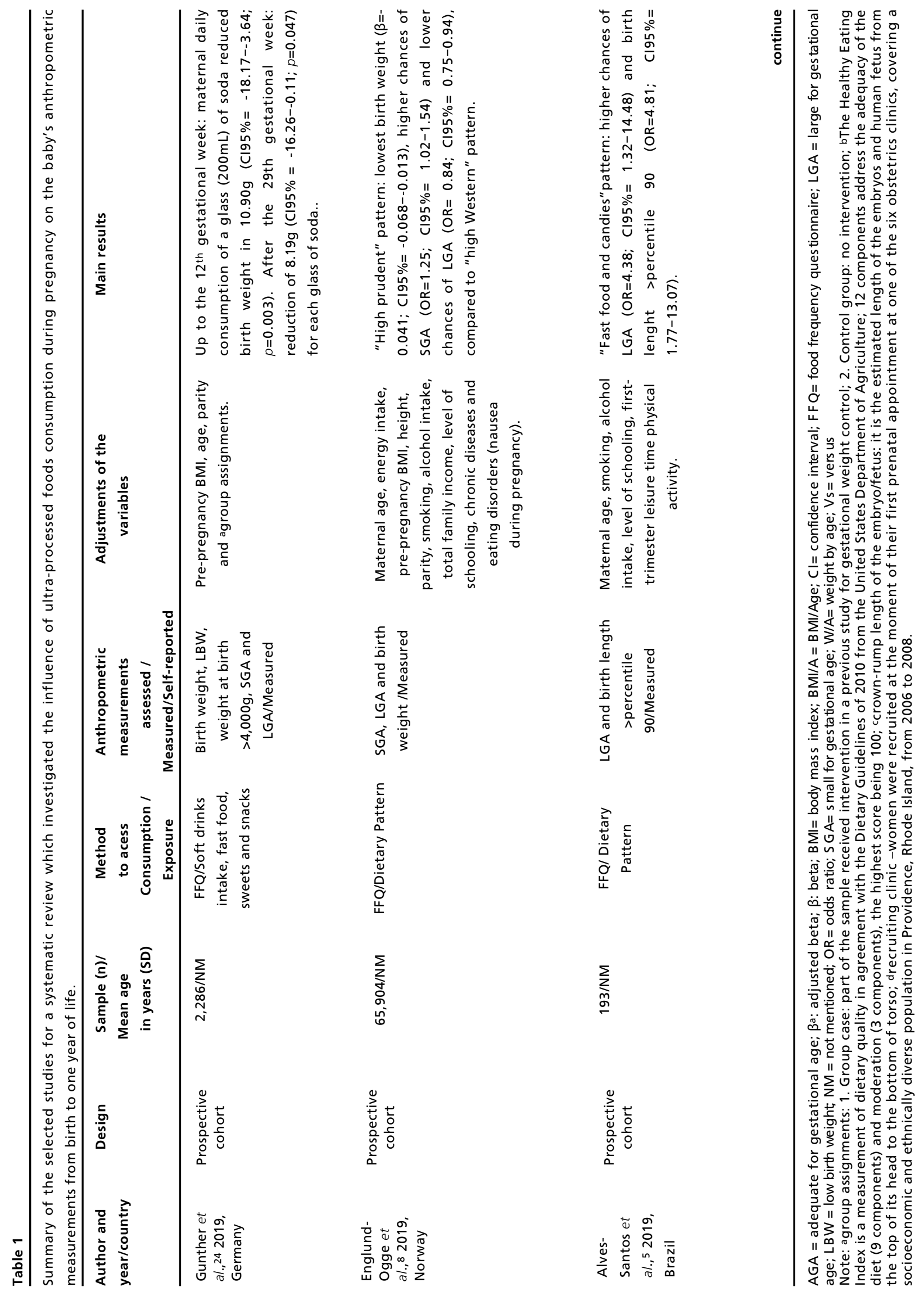




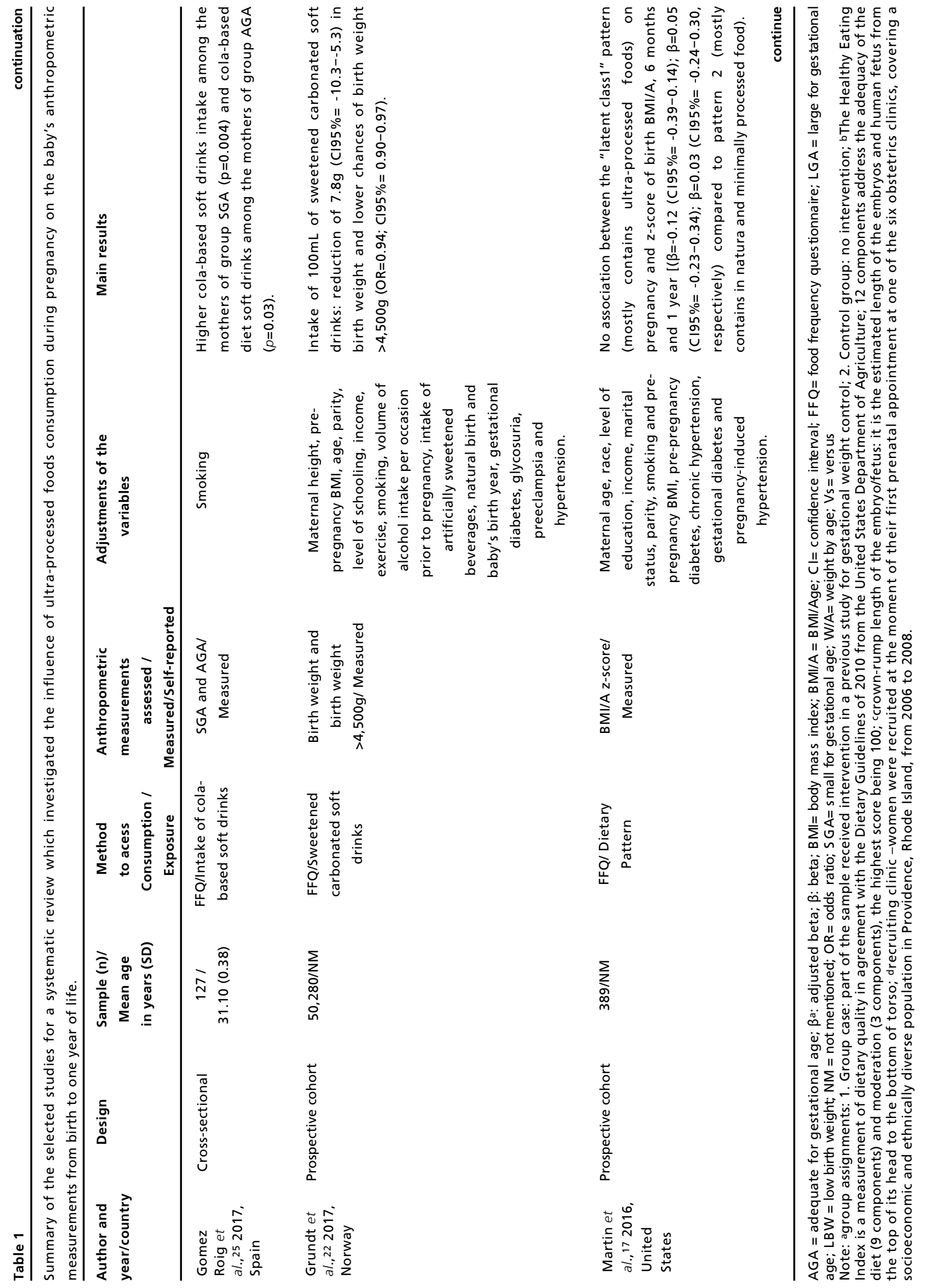




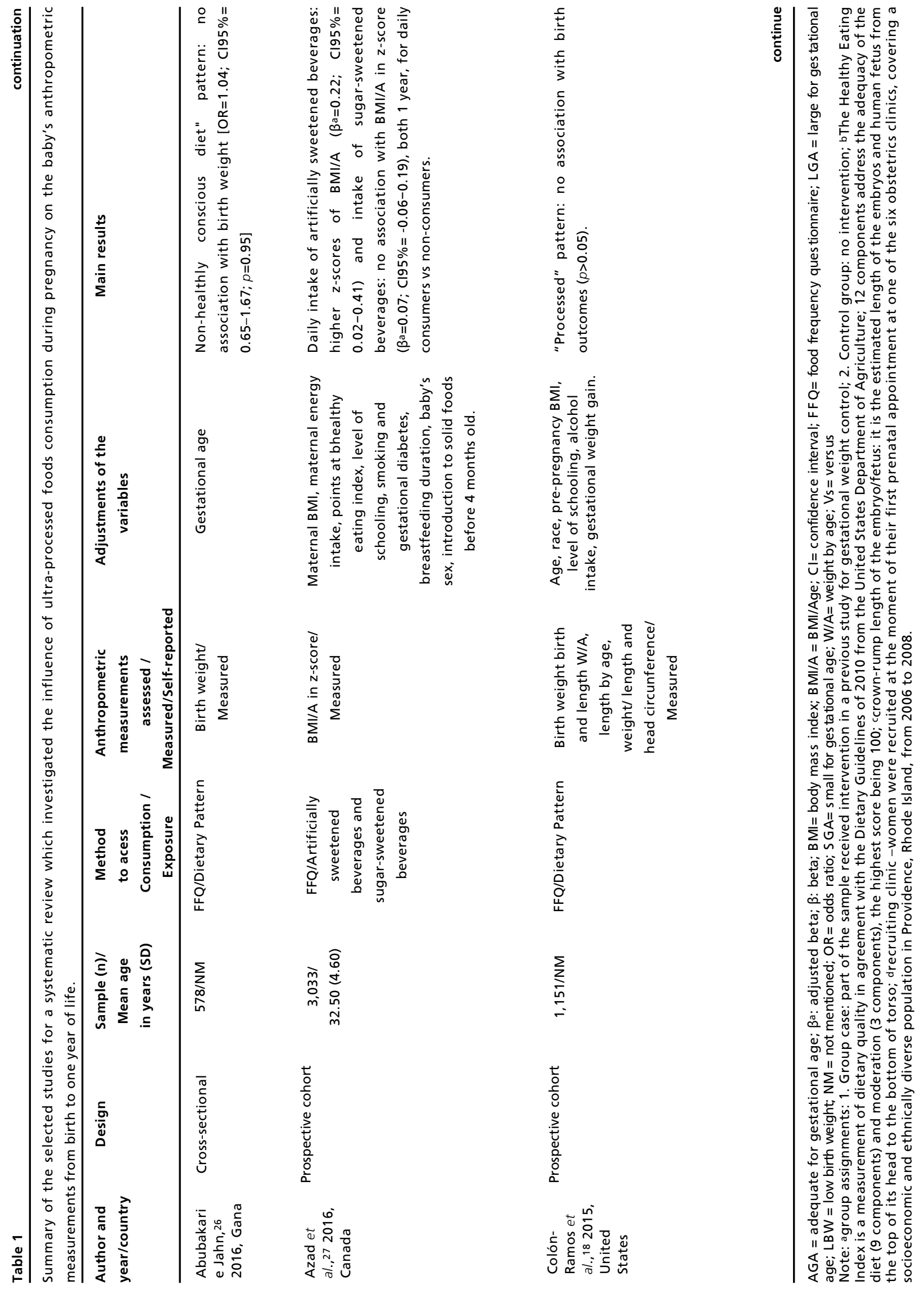




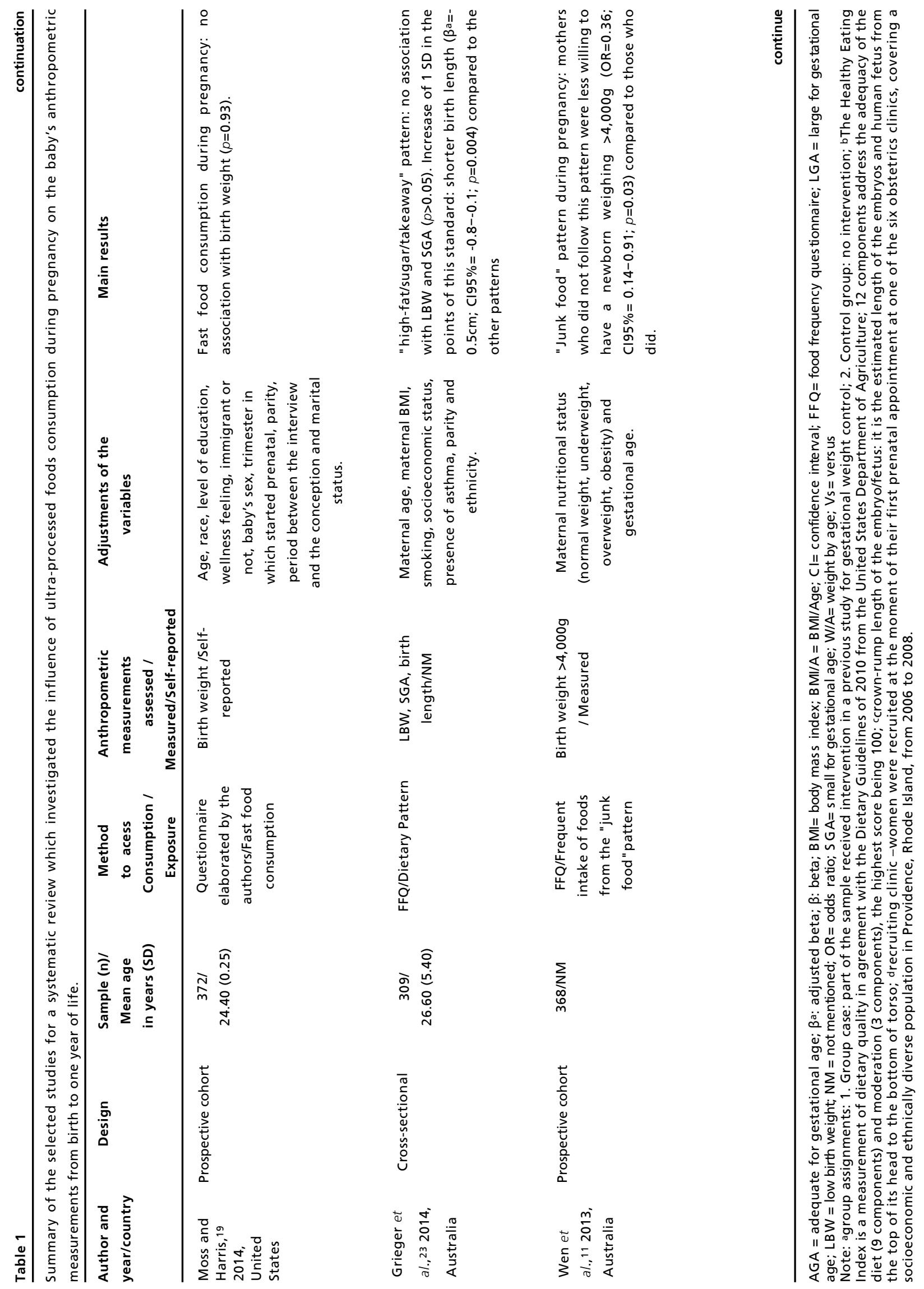



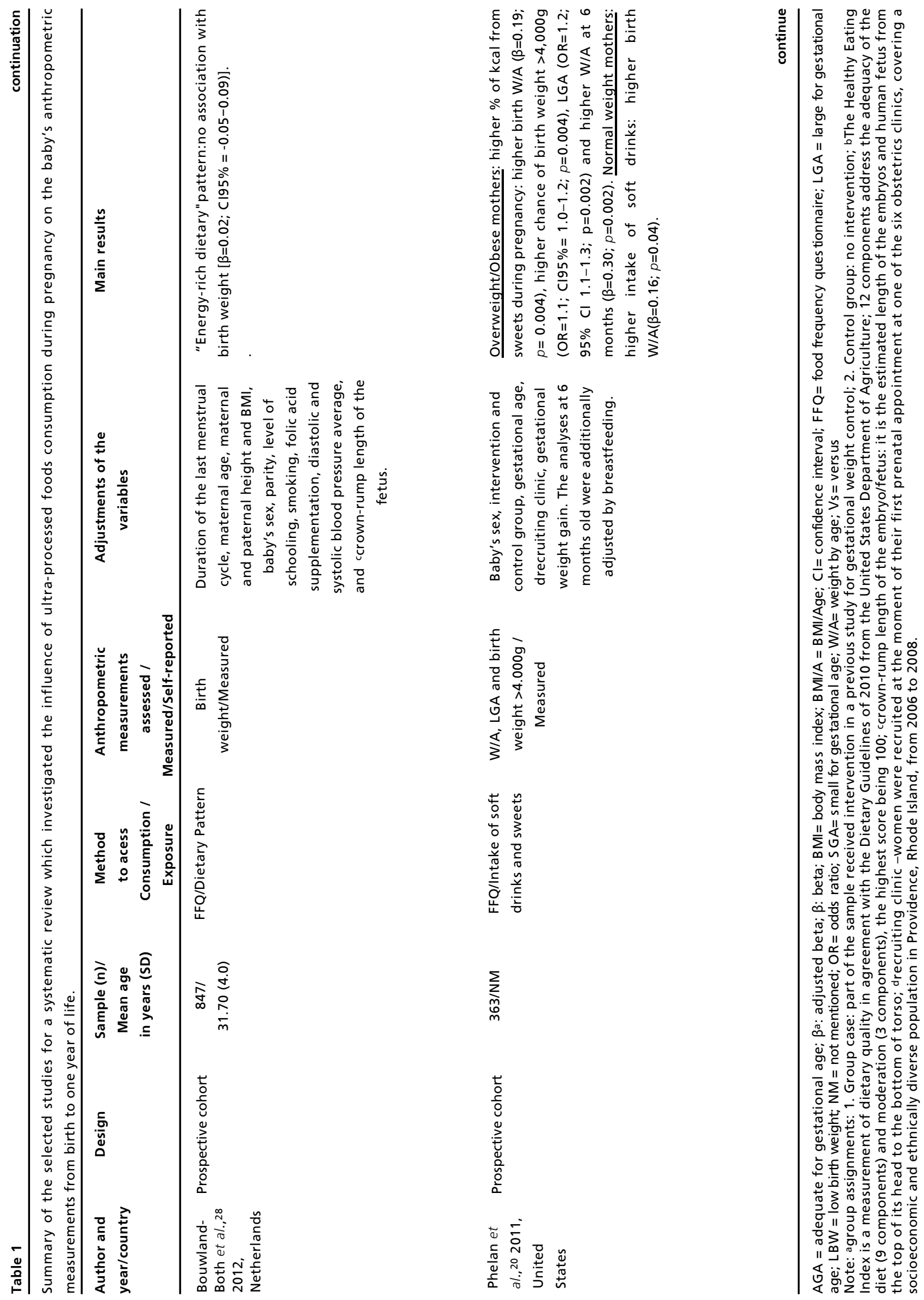


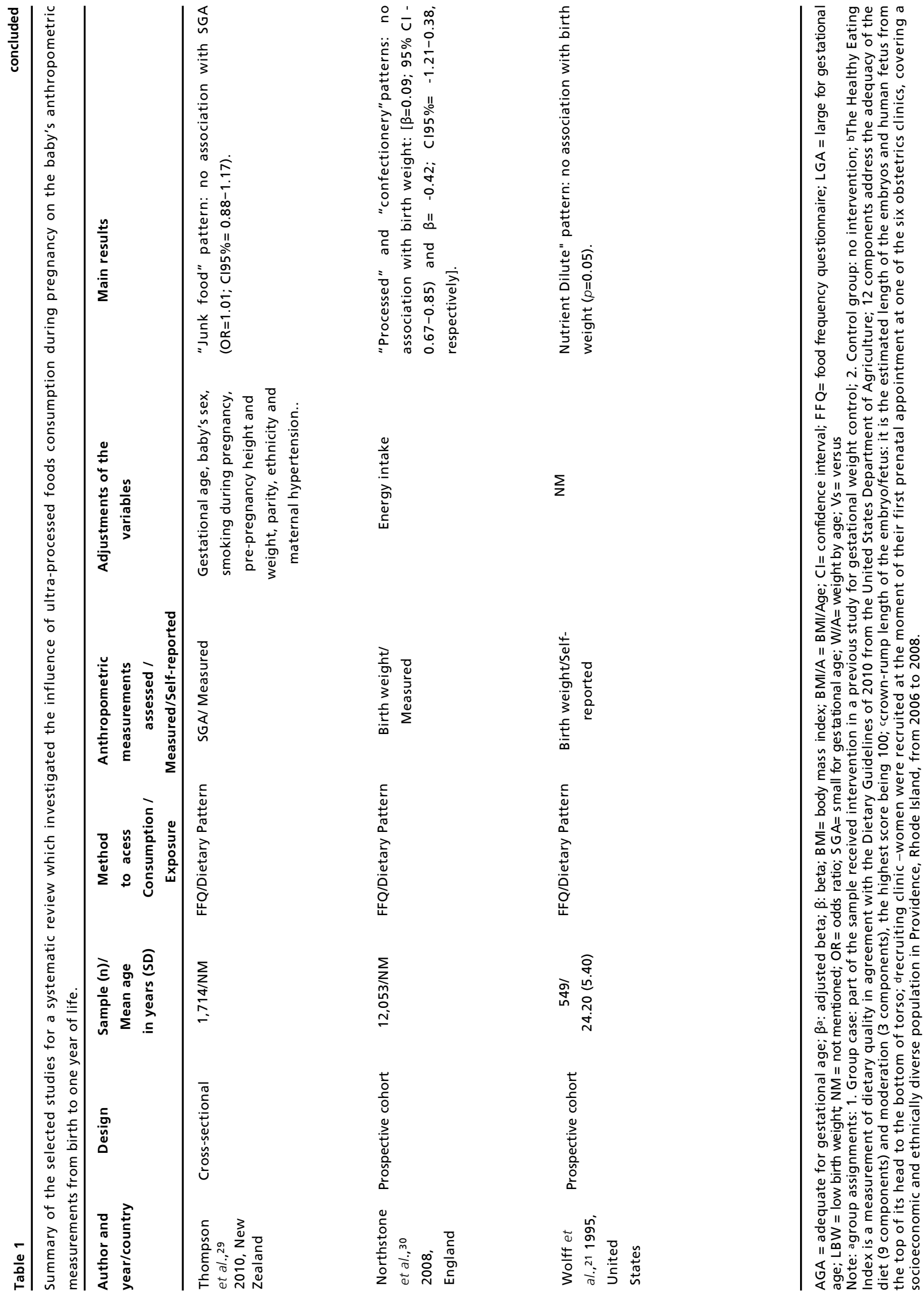


cially sweetened beverages and the BMI/A of the 1year-old baby (adjusted $\beta=0.22$; CI95\% $=0.02-0.41$ ) (Table 1). Phelan et al. ${ }^{20}$ described in a research of the same design, that in overweight/obese mothers, the highest percentage of calories from sweets during pregnancy was associated with higher birth W/A $(\beta=0.19 ; p=0.004)$ and at 6 months $(\beta=0.30$; $p=0.002)$, greater chance of birth weight $>4,000 \mathrm{~g}$ $[\mathrm{OR}=1.1 ; \mathrm{CI} 95 \%=1.0-1.2 ; p=0.004]$ and LGA babies $[\mathrm{OR}=1.2 ; \mathrm{CI} 95 \%=1.1-1.3 ; p=0.002]$ (Table 1).

In the longitudinal study by Wen et al.,11 mothers who did not follow a "junk food" diet pattern during pregnancy were less likely to have a newborn weighing $>4,000 \mathrm{~g}(\mathrm{OR}=0.36$; CI95\% $=0.14-0.91$; $p=0.03$ ) (Table 1), compared to those who followed such a pattern.

The inverse associations with the outcomes have been demonstrated in 4 studies.8,22,23,24 Grieger et al. 23 showed in a cross-sectional evaluation, that an increase of 1 standard deviation in the scores of the "high-fat/sugar/takeaway" pattern was associated with shorter birth length $(\beta a=-0.5 \mathrm{~cm} ; \mathrm{CI} 95 \%=-0.8-$ $-0.1 ; p=0.004)$. Likewise, Englund-Ogge et al. 8 noted in a prospective cohort, a greater chance of the birth of SGA babies (OR=1.25; CI95\%=1.02 - 1.54) among mothers belonging to the "high prudent" pattern compared to those of the "high western" pattern (Table 1).

In the longitudinal study by Gunther et al.,24 the daily consumption of a glass $(200 \mathrm{~mL})$ of soft drink by the pregnant woman, before or on the $12^{\text {th }}$ gestational week, reduced birth weight in $10.90 \mathrm{~g}$ $(\mathrm{CI} 95 \%=-18.17--3.64 ; p=0.003)$ and, after the 29 th week, the reduction was $8.19 \mathrm{~g}(\mathrm{CI} 95 \%=-16.26--$ $0.11 ; p=0.047)$ per glass of soft drink. In the research by Grundt et al.,22 the intake of $100 \mathrm{~mL}$ of soft drink was associated with a reduction of $7.8 \mathrm{~g}$ $(\mathrm{CI} 95 \%=-10.3--5.3)$ in birth weight and fewer chances of birth weight $>4,500 \mathrm{~g} \quad(\mathrm{OR}=0.94$; CI95\% $=0.90-0.97$ ) (Table 1).

Non-significant associations between the exposures and the outcomes were pointed out in eight studies that investigated the influence of ultraprocessed patterns in the baby's anthropometric measurements, in five cohorts 17,18,21,28,30 and three cross-sections 23,26,29 (Table 1).

Such associations were also highlighted in the studies by Gunther et al.24 in which soft drinks consumption by the mother was not associated with low birth weight (LBW), birth weight $>4,000 \mathrm{~g}$, SGA and LGA, and in the studies by Phelan et al.20 in which the intake of soft drinks was not associated with the child's W/A at six months, birth weight
$>4,000 \mathrm{~g}$ and LGA (data not presented in a table). In the study by Azad et al., 27 non-significant association between the exposure and the outcome was also observed between the intake of sugar-sweetened beverages and the BMI/A of the 1-year-old baby (adjusted $\beta=0.07 ;$ CI95\% $=-0.06-0.19$ ) (Table 1).

Gunther et al. 24 who investigated the consumption of "fast food", sweets and snacks, during pregnancy, observed that the intake of these foods did not influence with birth weight, LBW, birth weight $>4,000 \mathrm{~g}$, SGA and LGA, similar to Moss and Harris, 19 who did not find any associations between "fast food" consumption and birth weight $(p=0.93)$ (Table 1).

The main adjustment variables used in the analyses were maternal age, parity, smoking, level of schooling, pre-gestational BMI, race/ethnicity, baby's sex, gestational age, height, energy intake, alcohol intake, total family income, maternal BMI, gestational weight gain, marital status and breastfeeding (included in the studies which analysis was performed after the baby had been born).

Table 2 presents a summary of the associations (direct, inverse, and non-significant association between the exposure and the outcome) found in the articles that assess the influence of the consumption of ultra-processed foods during pregnancy on the baby's anthropometric measurements from birth to the first year.

In general, it is noted that non-significant association between the exposure and the outcome $(n=36)$ prevailed between the exposures (mostly ultraprocessed food pattern; soft drinks, artificially sweetened beverages and beverages sweetened with sugar; "fast food", "junk food", sweets and snacks) and the baby's anthropometric measurements.

Thirteen direct associations were found regarding the baby's anthropometric measurements: four when the exposure was ultra-processed dietary patterns; four when the association was with the consumption of soft drinks, artificially sweetened beverages and sugar-sweetened beverages and five when the explanatory variables were the consumption of "fast food", "junk food", sweets and snacks (Table 2).

Five inverse associations with the assessed outcomes were mentioned: two when the exposure was ultra-processed dietary patterns, and three when it was the consumption of soft drinks, artificially sweetened beverages and sugar-sweetened beverages (Table 2). 


\section{Discussion}

The present review showed that the majority of the studies that investigated mostly ultra-processed dietary patterns, the consumption of soft drinks, artificially sweetened beverages and sugar-sweetened beverages and "fast food", "junk food", sweets and snacks, found non-significant association with the baby's anthropometric measurements from birth to one year of life. This result contradicts the hypothesis of the authors of the present review that a high consumption of ultra-processed foods during pregnancy could lead to a greater occurrence of changes in the baby's anthropometric measurements and later, of the child, 3 considering the high energy density and low nutritional quality of those foods. 1 Some hypotheses have been postulated to explain such divergences.

First, it is important to highlight some methodological issues inherent to the studies assessed. Mothers with comorbidities such as gestational diabetes, hypertension, overweight/obesity, depression, and among others, were part of the samples under analysis. Although non-significant associations between the exposures and the outcomes were found in four 18,21,23,30 of the five studies $18,21,23,25,30$ that did not mention comorbidities, the presence of any disease or condition during pregnancy can promote an unfavorable gestational evolution, including an increased risk of birth of the newborns with weight deviations. 1

The main justification for not excluding these mothers from the sample was the use of these variables to adjust the final regression model $8,11,17,22,27-$ 29 and sensitivity analyses to examine the robustness of the results, ${ }^{17}$ which suffered minimal 22 or no interference 8 from the use of those variables.

Another methodological aspect needs to be considered, which may have influenced the nonsignificant associations between the exposures and the outcomes identified, concerns the lack of agreement regarding the moment of evaluation of food consumption among the studies. In addition to impairing the comparability of findings among the studies, such inconsistency may have interfered with the results, considering that pregnancy is a period marked by intense physiological, metabolic and endocrine changes. These are responsible for altering nutritional needs, food intake and nutritional maternal status, which are determinants in gestational weight gain, which is directly or indirectly associated with the newborn's and the child's health outcomes in the future. 6

Nevertheless, it is necessary to consider the cha- racteristics inherent to ultra-processed foods (such as high energy density, low nutritional value, high levels of sugars and caffeine - present mainly in cola-based soft drinks - in addition to fat and saturated fat), which can interfere with the gestational weight gain (including in women who already start pregnancy overweight) and, consequently, in the baby's anthropometric measurements, 5 which can justify the direct and inverse associations found in some studies.

In this sense, diet represents one of the main factors that influence pregnancy outcomes. An unhealthy diet consisting of ultra-processed foods, before and during pregnancy, can increase maternal body weight, increase the risk of birth for LGA babies and impact negatively on the mother and child's health in the short and long term. 5 Thus, in two recent studies, one national 5 and the other international, 8 "fast food and candies" showed a greater chance of LGA and birth length $>90^{\text {th }}$ percentile and the "high Western" pattern showed a greater chance of LGA.

However, in the case of cola-based soft drinks, the relationship between their consumption and the birth of SGA babies remains uncertain. ${ }^{25}$ Soft drinks are components of the pattern that provides energy and no specific nutrients. Its high intake can be accompanied by a lower intake of nutritious foods and this could explain the inverse association with birth weight. In addition, the role of soft drinks in this outcome needs to be better clarified, as the literature points out that the consumption of beverages containing sugar can have both an increasing and decreasing effect on birth weight. ${ }^{24}$

Another hypothesis that could justify the inverse association between the intake of sweetened carbonated soft drinks and birth weight and fewer chances of macrosomia would be the rapid sugar absorption provided by these beverages, resulting in glycemic spikes. If they occur frequently, they could induce oxidative stress, inflammation and microvascular endothelial dysfunction impairing blood flow through the placenta, reducing nutrition and fetal oxygenation. 22

In line with this hypothesis, another possible biochemical mechanism that could be associated would be the presence of pro-inflammatory nutrients in ultra-processed foods, such as fat and saturated fat that would limit the transfer of proper nutrients for the baby 23 through the placenta.

It is important to highlight the presence of mothers with comorbidities in the sample of eleven studies as a limitation of this review. In two of these, the treatment of these comorbidities was not 


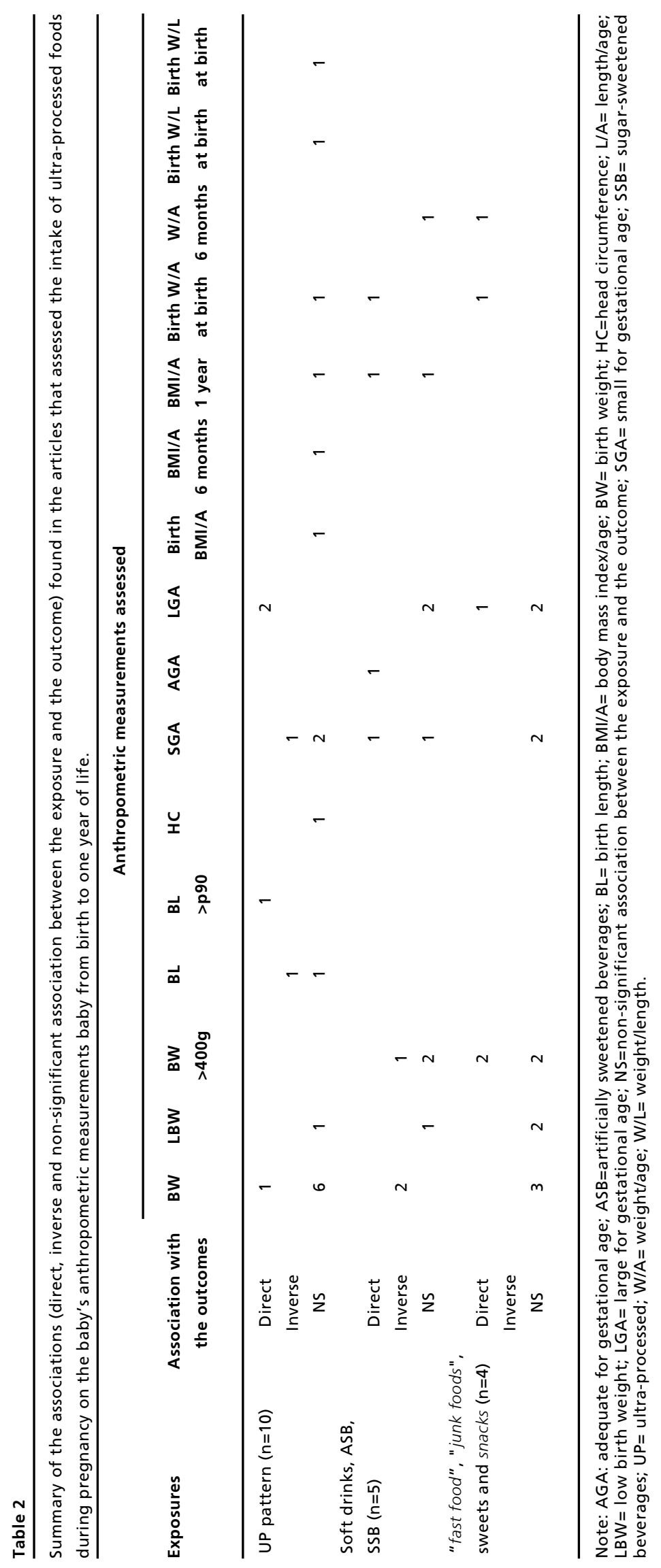

Rev. Bras. Saúde Mater. Infant., Recife, 21 (1): 9-26 jan-mar., 2021 
mentioned, so as not to interfere with the results obtained. Also, most of the studies lack information about the mothers' age. The literature points out that age is a factor that can interfere with food consumption and that it is difficult to change eating habits, even during pregnancy and, considering ultraprocessed foods, unhealthy dietary practices are more common among adolescents and young adults. 31 The lack of knowledge on healthy eating by pregnant young women is reflected in their food choices, which are influenced by factors such as increased appetite, "desire", marked taste, and the availability and convenience of food. 32

Finally, assessing food consumption of an individual or population is complex, due to its variability and the interaction between the various nutrients and food that compose it, in addition to the several possibilities of outcomes of this assessment, whether through dietary patterns, isolated food, food groups or analysis according to the degree of processing as proposed by NOVA.

NOVA is fairly recent and up to this moment, the authors are unaware of studies in the literature in which experts applied it to assess associations between the consumption of ultra-processed foods during pregnancy and the baby's anthropometric measurements. It is believed that this systematic review is the first to address the association between the consumption of ultra-processed foods during pregnancy and the baby's anthropometric measurements up to one year of life. Besides, it is noteworthy that a detailed analysis of the selected articles was carried out, concerning the investigated associations, the instruments used in the evaluation of food consumption, the moment to apply these instruments, sample representativeness and adjustment variables used in the studies to minimize confounding factors.

Most of the literature assessed did not show any influence of the consumption of ultra-processed foods during pregnancy on the baby's anthropometric measurements up to one year of life and pointed to a smaller number of direct and inverse associations between the exposures and analyzed outcomes. However, given the methodological diversity and complexity of the theme, further studies using a standardized food classification such as NOVA, are needed, so as to clarify the role of these ultra-processed products in the baby's anthropometric measurements.

\section{Acknowledgments}

This research was funded by Fundação de Amparo à Pesquisa do Estado de Minas Gerais (FAPEMIG) and National Council for Scientific and Technological Development (CNPq).

\section{Authors' contributions}

CM conducted the bibliographic review, conception, analysis and interpretation of the data, and final writing. RCVS participated in the bibliographic review, analysis and interpretation of data and critical review of intellectual content. LCS guided the research, analyzed the results critically, supported the writing, revised the manuscript and approved the final version of the article. 


\section{References}

1. Sotero AM, Cabral PC, Silva GAP. Fatores socioeconômicos, culturais e demográficos maternos associados ao padrão alimentar de lactentes. Rev Paul Pediatr. 2015; 33 (4): 445-52.

2. Brasil. Ministério da Saúde. Secretaria de Atenção à Saúde. Departamento de Atenção Básica. Guia Alimentar para a População Brasileira. $2^{\mathrm{a}}$ edição. Brasília, 2014. [acesso em 16 jul2020]. Disponível em: https://bvsms.saude.gov.br/ bvs/publicacoes/guia_alimentar_populacao_brasileira_2ed. pdf

3. Murphy MM, Stettler N, Smith KM, Reiss R. Associations of consumption of fruits and vegetables during pregnancy with infant birth weight or small for gestational age births: a systematic review of the literature. Int J Womens Health. 2014:6 899-912.

4. Brasil. Ministério da Economia. Instituto Brasileiro de Geografia e Estatística - IBGE Diretoria de Pesquisas. Coordenação de Trabalho e Rendimento.Pesquisa de Orçamentos Familiares 2017-2018. Avaliação Nutricional da Disponibilidade Domiciliar de Alimentos no Brasil. 61 p. Rio de Janeiro; 2020.

5. Alves-Santos NH, Cocate PG, Benaim C, Farias DR, Emmett PM, Kac G. J Acad Nutr Diet. 2019; 119 (9): 1439 51

6. Oliveira ACM, Pereira LA, Ferreira RC, Clemente APG Estado nutricional materno e sua associação com o peso ao nascer em gestações de alto risco. Ciênc Saúde Coletiva. 2018; 23(7): 2373-82.

7. Villar J, Ismail LC, Victora CG, Ohuma EO, Bertino E, Altman DG, Lambert A, Papageorghiou AT, Carvalho M, Jaffer YA, Gravett MG, Purwar M, Frederick IO, Noble AJ, Pang R, Barros FC, Chumlea C, Bhutta ZA, Kennedy SH International Fetal and Newborn Growth Consortium for the 21st Century (INTERGROWTH-21st). International standards for newborn weight, length, and head circumference by gestational age and sex: the Newborn CrossSectional Study of the INTERGROWTH-21st Project. Lancet. 2014; 384 (9946): 857-68.

8. Englund-Ögge L, Brantsæter AL, Juodakis J, Haugen M, Meltzer HM, Jacobsson B, Sengpiel V. Associations between maternal dietary patterns and infant birth weight, small and large for gestational age in the Norwegian Mother and Child Cohort Study. Eur J Clin Nutr. 2019; 73 (9): 1270-82.

9. Sassá AH, Higarashi IH, Bercini LO, Arruda DC, Marcon SS. Bebê de risco: acompanhando o crescimento infantil no primeiro ano de vida. Acta Paul Enferm. 2011; 24 (4): 5419.

10. Cunha AJLA, Leite AJM, Almeida IS. The pediatrician's role in the first thousand days of the child: the pursuit of healthy nutrition and development. J Pediatr. 2015; 91 (6 Suppl 1): S44-S51.

11. Wen LM, Simpson JM, Rissel C, Baur LA.Maternal "junk food" diet during pregnancy as a predictor of high birthweight: findings from the healthy beginnings trial. Birth. 2013; 40 (1): 46-51.
12. Galvão TF, Pereira MG. Revisões sistemáticas da literature: passos para sua elaboração. Epidemiol Serv Saude. 2014; 23(1): 183-4.

13. World Health Organization (WHO), The United Nations Children's Fund (Unicef). Low Birthweight: Country, regional and global estimates. UNICEF, New York, 2004 [acesso 29 ago 2020]. Disponível em:https://apps.who.int/ iris/bitstream/handle/10665/43184/9280638327.pdf?sequen $\mathrm{ce}=1$.

14. Brasil. Ministério da Saúde. Secretaria de Atenção à Saúde. Departamento de Ações Programáticas Estratégicas. Gestação de alto risco: manual técnico / Ministério da Saúde, Secretaria de Atenção à Saúde, Departamento de Ações Programáticas Estratégicas. - 5. ed. - Brasília : Editora do Ministério da Saúde, 2012. 302 p. - (Série A Normas e Manuais Técnicos).[acesso em 29 ago2020]. Disponível em: http://bvsms.saude.gov.br/bvs/publicacoes/manual_tecnico_gestacao_alto_risco.pdf.

15. Byrt T. How good is that agreement? [letter]. Epidemiol. 1996; 7 (5): 561.

16. Brasil. Ministério da Saúde.Secretaria de Ciência, Tecnologia e Insumos Estratégicos. Departamento de Ciência e Tecnologia. Diretrizes metodológicas: elaboração de revisão sistemática e metanálise de estudos observacionais comparativos sobre fatores de risco e prognóstico. 132 p. Brasília. Ministério da Saúde. 2014.

17. Martin CL, Siega-Riz AM, Sotres-Alvarez D, Robinson WR, Daniels JL, Perrin EM, Stuebe AM. Maternal Dietary Patterns during Pregnancy Are Associated with Child Growth in the First 3 Years of Life. J Nutr. 2016; 146 (11): 2281-8.

18. Colón-Ramos U, Racette SB, Ganiban J, Nguyen TG Kocak M, Carroll KN, Völgyi E, Tylavsky FA. Association between Dietary Patterns during Pregnancy and Birth Size Measures in a Diverse Population in Southern US. Nutrients. 2015. 16; 7 (2): 1318-32.

19. Moss JL, Harris KM. Impact of maternal and paternal preconception health on birth outcomes using prospective couples' data in Add Health. Arch Gynecol Obstet. 2015; 291 (2): 287-98.

20. Phelan S, Hart C, Phipps M, Abrams B, Schaffner A, Adams A, Wing R. Behaviors during pregnancy impact offspring obesity risk. Exp Diabetes Res. 2011; 2011: 985139.

21. Wolff CB, Wolff HK. Maternal eating patterns and birth weight of Mexican American infants. Nutr Health. 1995; 10 (2): 121-34.

22. Grundt JH, Eide GE, Brantsaeter AL, Haugen M, Markestad T. Is consumption of sugar-sweetened soft drinks during pregnancy associated with birth weight? Matern Child Nutr. 2017; 13 (4): e12405.

23. Grieger JA, Grzeskowiak LE, Clifton VL.Preconception dietary patterns in human pregnancies are associated with preterm delivery. J Nutr. 2014; 144 (7): 1075-80. 
24. Günther J, Hoffmann J, Spies M, Meyer D, Kunath J, Stecher L, Rosenfeld E, Kick L, Rauh K, Hauner H.Associations between the Prenatal Diet and Neonatal Outcomes-A Secondary Analysis of the ClusterRandomised GeliS Trial. Nutrients. 2019. 13; 11 (8). pii: E1889.

25. Gómez Roig MD, Mazarico E, Ferrero S, Montejo R, Ibáñez L, Grima F, Vela A. Differences in dietary and lifestyle habits between pregnant women with small fetuses and appropriate-for-gestational-age fetuses. J Obstet Gynaecol Res. 2017; 43 (7): 1145-51.

26. Abubakari A, Jahn A. Maternal Dietary Patterns and Practices and Birth Weight in Northern Ghana.PLoS One. 2016. 9; 11 (9): e0162285.

27. Azad MB, Sharma AK, Souza RJ, Dolinsky VW, Becker AB, Mandhane PJ, Turvey SE, Subbarao P, Lefebvre DL, Sears MR. Association between artificially sweetened beverage consumption during pregnancy and infant bodymass index. JAMA Pediatr. 2016; 170 (7): 662-70.

28. Bouwland-Both MI, Steegers-Theunissen RPM, Vujkovic M, Lesaffre EMEH, Mook-Kanamori DO, Hofman A, Lindemans J, Russcher H, Jaddoe VWV, Steegers EAP. A periconceptional energy-rich dietary pattern is associated with early fetal growth: the Generation R study. BJOG. 2013; 120 (4): 435-45.

Received on July 16, 2020

Final version presented on September 15, 2020

Approved on December 4, 2020
29. Thompson JM, Wall C, Becroft DM, Robinson E, Wild CJ, Mitchell EA. Maternal dietary patterns in pregnancy and the association with small-for-gestational-age infants. Br J Nutr. 2010; 103 (11): 1665-73.

30. Northstone K, Ness AR, Emmett PM, Rogers IS. Adjusting for energy intake in dietary pattern investigations using principal components analysis. Eur J Clin Nutr.2008; 62 (7): 931-8.

31. Brito ACD, Abreu DAS, Cabral NAL, Silva MB, Gomes RS, Ribeiro VS. Consumo de frutas, verduras e legumes por gestantes adolescentes. Rev Bras Promoç Saúde. 2016; 29 (4): 480-9.

32. Barros DC, Pereira RA, Gama SGN, Leal MC. O consumo alimentar de gestantes adolescentes no Município do Rio de Janeiro. Cad Saúde Pública. 2004; 20 (Sup1): S121S129. 